\title{
Dissipative superconducting state of non-equilibrium nanowires
}

\author{
Yu Chen ${ }^{1,2}$, Yen-Hsiang Lin ${ }^{2,3}$, Stephen D. Snyder2†, Allen M. Goldman ${ }^{2 \star}$ and Alex Kamenev ${ }^{2,4}$
}

\begin{abstract}
The ability to carry electric current with zero dissipation is the hallmark of superconductivity'. This very property makes possible such applications ranging from magnetic resonance imaging machines to Large Hadron Collider magnets. But is it indeed the case that superconducting order is incompatible with dissipation? One notable exception, known as vortex flow, takes place in high magnetic fields ${ }^{2}$. Here we report the observation of dissipative superconductivity in far more basic configurations: superconducting nanowires with superconducting leads. We provide evidence that in such systems, normal current may flow in the presence of superconducting order throughout the wire. The phenomenon is attributed to the formation of a non-equilibrium state, where superconductivity coexists with dissipation, mediated by the so-called Andreev quasiparticles. Besides the promise for applications such as single-photon detectors ${ }^{3}$, the effect is a vivid example of a controllable non-equilibrium state of a quantum liquid. Thus our findings provide an accessible generic platform to investigate conceptual problems of out-of-equilibrium quantum systems.
\end{abstract}

With applications ranging from infrared detectors ${ }^{4}$ to prototypical qubits ${ }^{5,6}$, superconducting nanocircuitry has emerged in recent years as a fascinating area of research. Its fundamental significance lies in, for example, phase-sensitive studies of pairing mechanisms in novel superconductors ${ }^{7,8}$ and access to a wealth of non-equilibrium quantum phenomena ${ }^{9}$. Key elements of such circuitry-superconducting nanowires-are known to be susceptible to strong fluctuations. Their most spectacular manifestations are phase slips of the superconducting order parameter, which lead to dissipation within a nominally dissipationless superconducting state $\mathrm{e}^{10-12}$. Observing and studying such dissipative superconductivity has turned out to be a challenge. The culprits are non-equilibrium quasiparticles massively generated by phase slips. If not removed efficiently, they tend to overheat the nanowire, driving it into the normal state. For example, thin MoGe (refs 13,14 ) and $\mathrm{Al}$ wires ${ }^{15,16}$ seem to be switched into the normal state by a single phase slip. The return to the superconducting state requires a significant decrease of the drive current, leading to hysteretic $I-V$ characteristics.

In the experiments reported here we overcome the excess heating by an improved fabrication process (Methods). Electrically transparent interfaces between the wire and the leads allow a fast escape of non-equilibrium quasiparticles into the environment. By choosing $\mathrm{Zn}$ as the growth material ${ }^{17}$, we are able to fabricate quasi-
1D wires whose length $L$ is significantly shorter than the inelastic relaxation length $L_{\text {in }}$, yet much longer than the coherence length $\xi \approx 250 \mathrm{~nm}$. These bring us to a situation in which quasiparticles form a peculiar non-equilibrium distribution, governed by Andreev reflections from the boundaries with the superconducting leads. As a result, we observe a non-hysteretic dissipative state, which still exhibits distinct superconducting features such as a supercurrent and a sensitivity to weak magnetic fields.

In Fig. 1a, we show $I-V$ characteristics of sample A, measured at temperatures from 50 to $750 \mathrm{mK}$ in a well-filtered dilution refrigerator (Methods). Over a range of currents, flanked by the bottom and top threshold values $I_{\mathrm{b}}<I<I_{\mathrm{t}}$, the voltage across the nanowire exhibits a nearly flat plateau at $V_{0}=52.5 \pm 1.2 \mu \mathrm{V}$ (for $T \lesssim 450 \mathrm{mK}$ ). This indicates a peculiar dissipative state that is distinctly different from the normal state. It is important to note that both $I_{\mathrm{t}}$ and $I_{\mathrm{b}}$ are factors of 30-50 smaller than the estimated depairing critical current of the wire ${ }^{18}$. Collecting $I_{\mathrm{b}}$ and $I_{\mathrm{t}}$ together with $I_{c}$, where the system becomes normal, we construct a temperature-current phase diagram, shown in Fig. 1b. It shows that as the temperature increases, the voltage plateau is compressed and eventually disappears at approximately $650 \mathrm{mK}$. This temperature dependence resembles that of the superconducting order parameter, suggesting that the dissipative voltage plateau state is associated with the superconducting order.

Performing measurements on different devices, we found a remarkable universality associated with the voltage plateau. In Fig. $1 \mathrm{c}-\mathrm{f}$, we compare the $I-V$ characteristics of sample A with two other $\mathrm{Zn}$ samples, $\mathrm{B}$ and $\mathrm{C}$, as well as an $\mathrm{Al}$ sample $\mathrm{D}$, all measured at $450 \mathrm{mK}$. These samples differ from sample A both in their geometry and normal-state resistance, $R_{\mathrm{n}}$, and were measured in a different weakly filtered refrigerator. Despite some smearing due to unfiltered noise, the plateau voltage $V_{0}$ remains almost unchanged for all the $\mathrm{Zn}$ wires. The only exception is $\mathrm{Al}$ sample $\mathrm{D}$, with the voltage plateau at $V_{0} \simeq 93.2 \pm 1.3 \mu \mathrm{V}$. Rescaling this voltage with the Bardeen-Cooper-Schrieffer superconducting gap $2 \Delta_{0} \approx 3.52 T_{\mathrm{c}}$, we found that the plateaux in all samples fall close to the same universal line $e V_{0} / \Delta_{0}=0.43 \pm 0.05$ (the ratio for all samples is listed in the Supplementary Methods).

Another remarkable feature of the voltage plateau state is its onset through a region of stochastic bistability. It is revealed by time-domain measurements, with the voltage measured using a repetition rate of $3 \mathrm{~Hz}$ under a sustained constant current. In Fig. 2a we show a time trace of the measured voltage at $I=1.95 \mu \mathrm{A}$ and $T=50 \mathrm{mK}$. The system exhibits random switching between the

${ }^{1}$ Department of Physics, University of California-Santa Barbara, Santa Barbara, California 93106, USA, ${ }^{2}$ School of Physics and Astronomy, University of Minnesota, Minneapolis, Minnesota 55455, USA, ${ }^{3}$ Department of Physics, University of Michigan-Ann Arbor, Ann Arbor, Michigan 48109, USA, ${ }^{4}$ William I. Fine Theoretical Physics Institute, University of Minnesota, Minneapolis, Minnesota 55455, USA. †Present address: Intel Corp., Portland, Oregon 97124, USA. *e-mail: goldman@physics.umn.edu 
a

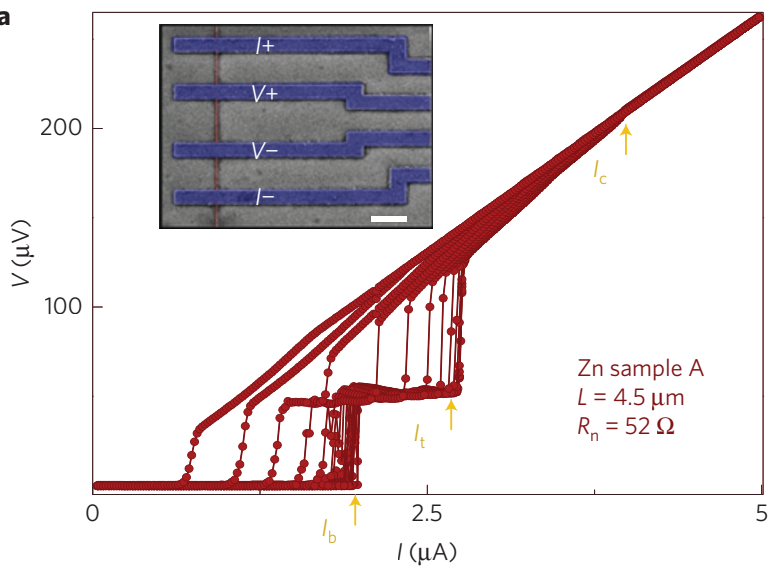

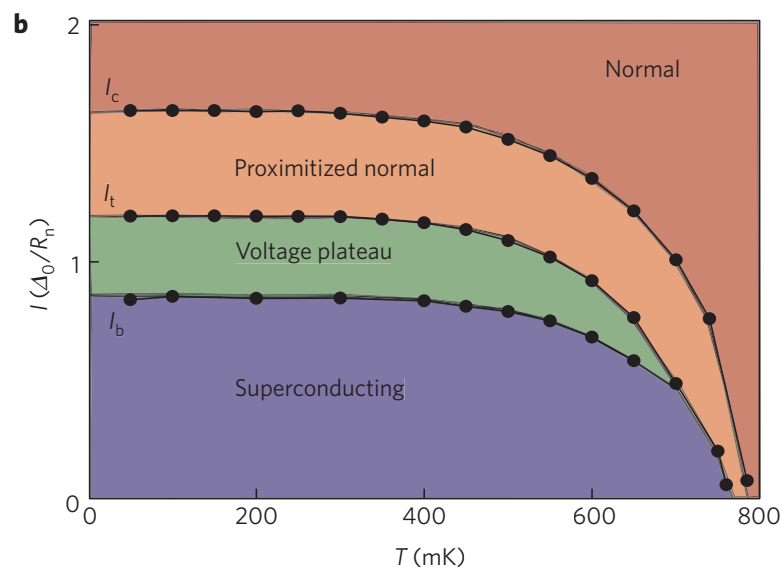

c e

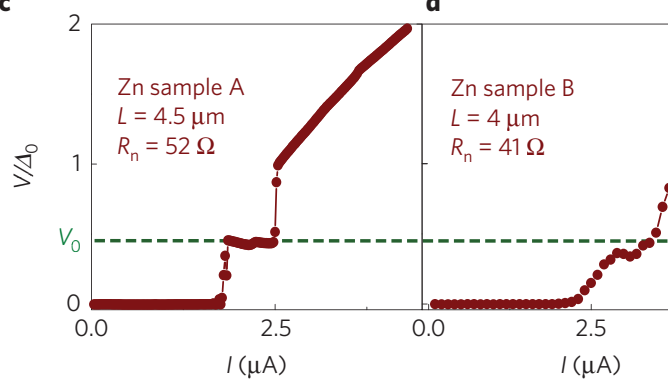

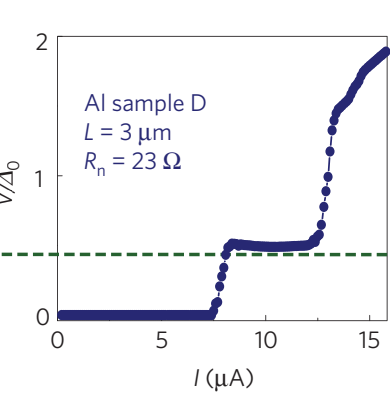

Figure 1 | Experimental observation of the voltage plateau. a, The $I-V$ characteristics of sample $A$, at temperatures from $50 \mathrm{mK}$ to $750 \mathrm{mK}$ (right to left) at intervals of $50 \mathrm{mK}$. The voltage plateau with $V_{0} \sim 52 \mu \mathrm{V}$ is visible between the bottom and top threshold currents $l_{\mathrm{b}}$ and $l_{\mathrm{t}}$. The inset shows a scanning electron microscope false colour image of the sample. Scale bar, $2 \mu \mathrm{m}$. $\mathbf{b}$, The temperature-current phase diagram, showing the voltage plateau existing at $T \lesssim 650 \mathrm{mK}$. c-f,$I-V$ characteristics of $\mathrm{Zn}$ samples A-C and Al sample $\mathrm{D}$, respectively, measured at $450 \mathrm{mK}$ with length, $L$, and normal-state resistance, $R_{\mathrm{n}}$ as indicated. Sample A was measured in the well-filtered cryostat, whereas samples B-D were measured in the weakly filtered cryostat. The voltage axes are scaled to the Bardeen-Cooper-Schrieffer energy gap $2 \Delta_{0}=3.52 T_{\mathrm{c}}$ for each sample. The voltage plateaux of all samples collapse onto $V_{0} / \Delta_{0} \sim 0.43 \pm 0.05$, providing the evidence for the universality of the plateau state.

superconducting and voltage plateau states with a characteristic timescale of a few seconds, indicating an intrinsic bistability. To quantify the stability of the two competing states we define lifetimes $\tau_{\mathrm{sc}}$ and $\tau_{\mathrm{vp}}$ as the averaged residence times in the superconducting and the voltage plateau states respectively. Figure $2 \mathrm{~b}$ shows the dependence of the lifetimes on the applied current throughout the transition range. Increasing the current leads to an exponential growth of the voltage plateau lifetime $\tau_{\mathrm{vp}}$ and the reduction of the superconducting lifetime $\tau_{\mathrm{sc}}$, albeit at a smaller rate. It is worth mentioning that the two lifetimes are almost independent of temperature until $T \sim 400 \mathrm{mK}$, above which $\tau_{\mathrm{vp}}$ increases and $\tau_{\mathrm{sc}}$ decreases exponentially (see Supplementary Methods).

The observed dissipative state exhibits a counter-intuitive magnetic field dependence. One could expect that the magnetic field suppresses superconductivity, thus decreasing $\tau_{\text {sc }}$ and possibly increasing $\tau_{\mathrm{vs}}$. In fact, the exact opposite happens. As shown in Fig. 2c, a magnetic field of merely a few gauss stabilizes the superconducting state, increasing its lifetime by more than an order of magnitude and simultaneously decreasing the voltage plateau lifetime by two orders of magnitude. This behaviour is consistently observed through the entire transition range of currents.

The enhancement of superconductivity by magnetic field is even more apparent by inspecting the $I-V$ characteristics at different fields (Fig. 2d). It is evident that the field shifts the bottom critical current $I_{\mathrm{b}}$ to higher values, stabilizing the superconducting state. Such a stabilization is in fact a result of the suppression of the voltage plateau state. This is best seen in the critical current versus magnetic field phase diagram (Fig. 2e). The range of currents supporting the voltage plateau decreases rapidly from below until the plateau collapses at $19 \mathrm{G}$. Correspondingly the phase space of the superconducting state expands. We thus observe a rather counterintuitive non-equilibrium phenomenon: keeping the current within the voltage plateau regime and increasing the magnetic field brings the system from the dissipative into the superconducting state. This is consistent with the reported magnetic-field-induced superconductivity and anti-proximity effect ${ }^{19-22}$. It is now apparent that the magnetic-field-induced superconductivity originates with the collapse of the voltage plateau state, providing an intriguing connection between the two effects.

It is crucial to distinguish the observed voltage plateau state from other phenomena. It is different from phase slip centres, seen in long superconducting whiskers and characterized by a constant differential resistance ${ }^{18,23}$, as opposed to a constant voltage. The plateau cannot be a giant Shapiro step ${ }^{24,25}$, caused by leaking highfrequency noise. Indeed, $\mathrm{Zn}$ and $\mathrm{Al}$ samples have different $V_{0}$ values, requiring noise of very different frequencies within the same measurement apparatus. It also cannot be attributed to a running state of an underdamped Josephson junction ${ }^{26}$. The underdamped regime would require a capacitance orders of magnitude larger than that of our system (Supplementary Methods). External capacitance is also excluded by the fact that the same results were obtained in two refrigerators with very different circuitry. Moreover, contrary to the observed plateau, voltage across an underdamped junction is expected to grow with the increased current bias.

Key to understanding these observations are non-equilibrium quasiparticles generated by phase slips ${ }^{18}$. In our samples (in contrast 

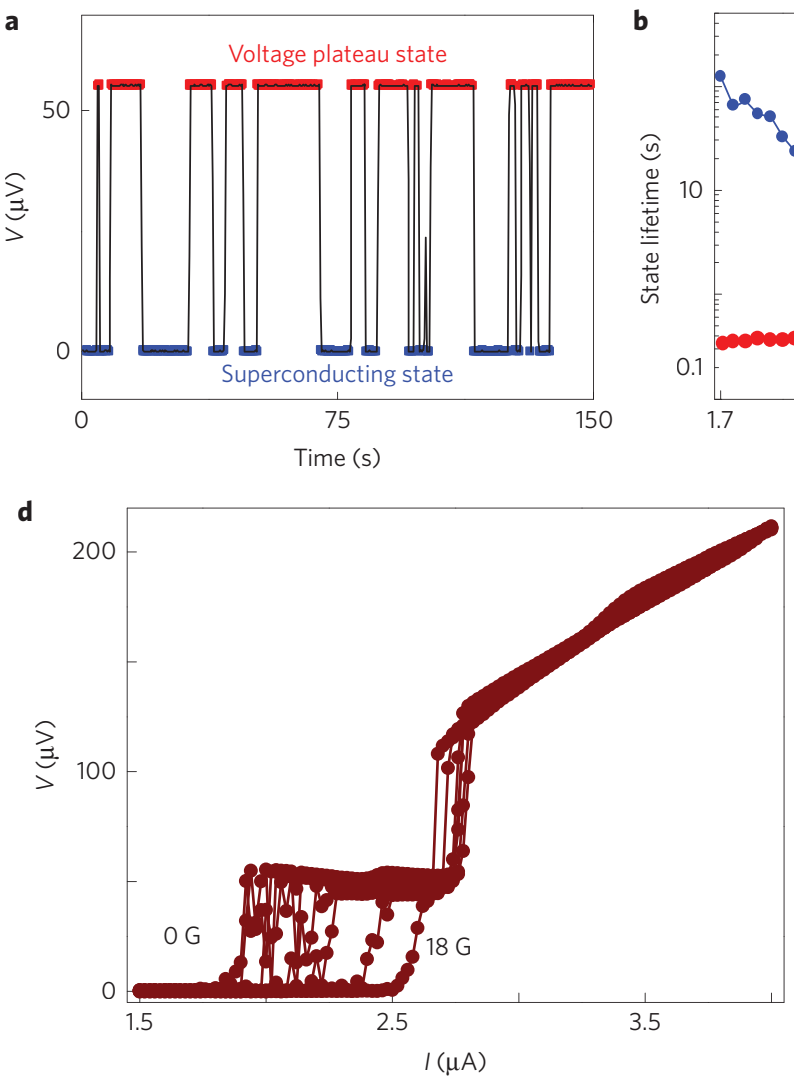
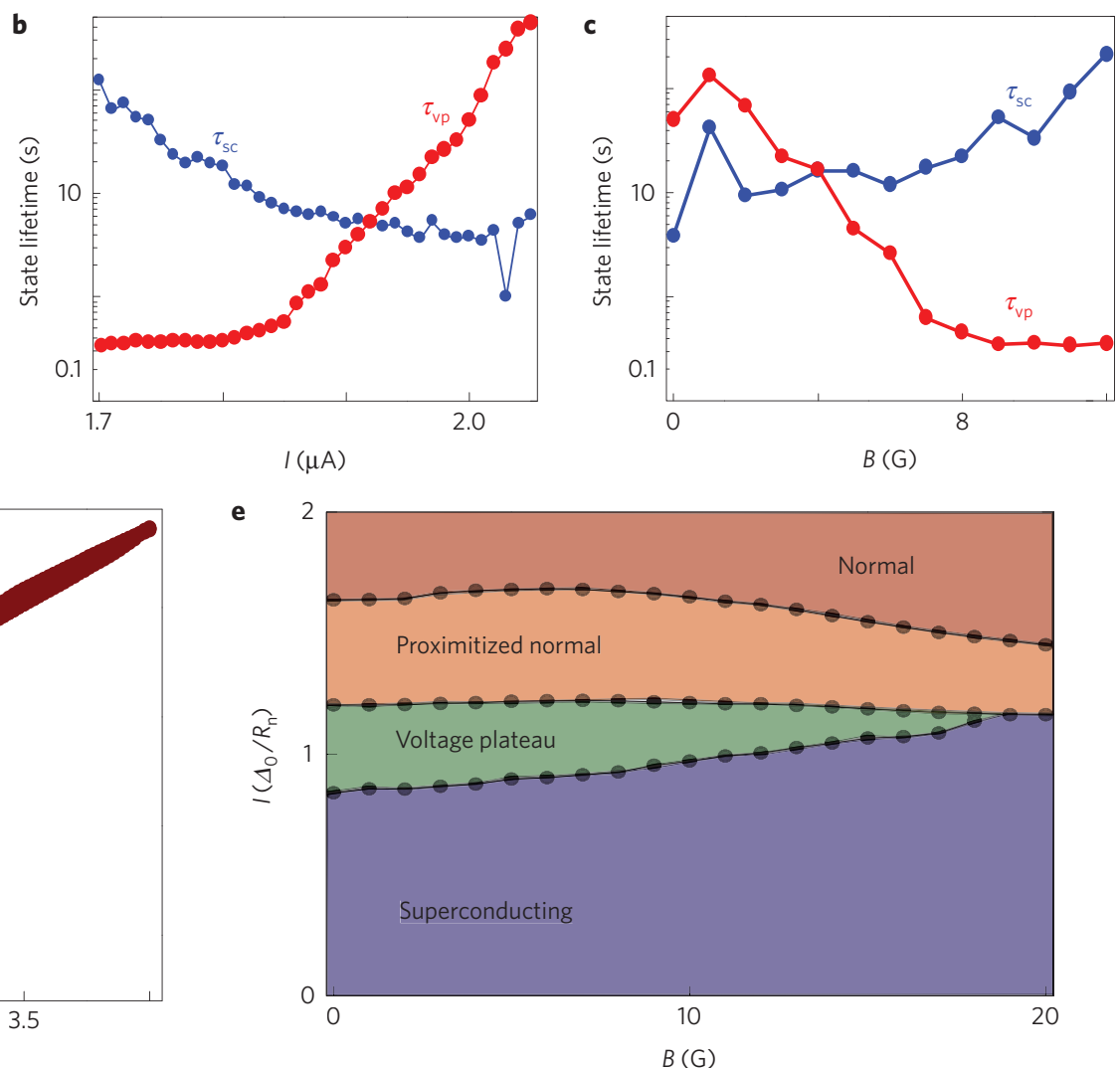

Figure $\mathbf{2}$ | The onset through bistability and the magnetic response of the voltage plateau. a, The real-time evolution of the voltage for sample A held at a fixed current of $1.95 \mu \mathrm{A}$ and a temperature of $50 \mathrm{mK}$. The system undergoes stochastic switching between the superconducting and the voltage plateau states. b. Average lifetimes of the superconducting $\left(\tau_{\mathrm{sc}}\right)$ and voltage plateau $\left(\tau_{\mathrm{vp}}\right)$ states at elevated currents. The transition is accomplished by suppressing the superconducting state and stabilizing the voltage plateau state. c, Average lifetimes as functions of the perpendicular magnetic field. A magnetic field of only a few gauss suppresses the lifetime of the voltage plateau state by over two orders of magnitude. $\mathbf{d}, I-V$ characteristics at magnetic fields from 0 to $18 \mathrm{G}$ in intervals of $2 \mathrm{G}$. The bottom critical current $\left(\mathrm{I}_{\mathrm{b}}\right)$ is seen to increase from $\sim 1.8 \mu \mathrm{A}$ to $\sim 2.5 \mu \mathrm{A}$. e, Magnetic field-current phase diagram shows the high sensitivity of the voltage plateau to magnetic field. The plateau disappears at $19 \mathrm{G}$.

with previous studies ${ }^{13-16,18}$ ) the inelastic relaxation length $L_{\text {in }}$ exceeds the wire length ${ }^{17}$, allowing the quasiparticles to spread over the entire wire. At the interfaces with the leads the quasiparticles experience Andreev reflections, which mix particles and holes, as shown in Fig. 3. This leads to quasiparticle diffusion over energy ${ }^{27}$, resulting in a peculiar non-equilibrium distribution. Because of the self-consistency relation, such a non-equilibrium distribution suppresses the order parameter $\Delta$ inside the wire (relative to its equilibrium value $\Delta_{0}$ ). Although the quasiparticles are far from equilibrium, the order parameter is fixed to its local self-consistent value everywhere apart from a distance $\sim \xi$ around the phase slip. The condensate chemical potential $\mu$, given by the Josephson relation $\mu=\hbar\left\langle\partial_{\mathrm{t}} \phi\right\rangle / 2$, thus exhibits a discontinuity $e V$ at the phase slip location ${ }^{18}$. Because the leads absorb high-energy quasiparticles, the concentration of the latter is largest in the centre, pinning the phase slip to the midpoint of the wire. For phase slips to occur, the voltage must exceed the energy gap (Fig. 3), namely, $e V_{0} \approx 2 \Delta$. In this case the train of phase slips permanently suppresses the order parameter in the narrow middle region, self-propelling the dissipative state (that is, in such a self-consistent dynamical regime the rate of phase slips is not exponentially small).

For a quantitative description (see Supplementary Methods for the full calculation), it is convenient to parametrize the quasiparticle distribution function $F(\epsilon, x)=1-2 n(\epsilon, x)$ by its longitudinal, $F_{\mathrm{L}}$, and transverse, $F_{\mathrm{T}}$, components, which are its odd and even parts $F_{\mathrm{L} / \mathrm{T}}(\epsilon, x)=F\left(\epsilon-\mu_{1,2}, x\right) \mp F\left(-\epsilon+\mu_{1,2}, x\right)$ with respect to the two chemical potentials $\mu_{1,2}= \pm e V / 2$. At the boundaries with the leads $x=x_{1,2}(\epsilon)$ and the energy window $\Delta<\left|\epsilon-\mu_{1,2}\right|<\Delta_{0}$ they obey Andreev boundary conditions:

$$
\left.F_{\mathrm{T}}(\epsilon, x)\right|_{x=x_{a}(\epsilon)}=0 ;\left.\quad \partial_{x} F_{\mathrm{L}}(\epsilon, x)\right|_{x=x_{a}(\epsilon)}=0
$$

In the absence of inelastic relaxation the continuity relation (known also as the Usadel equation $\left.{ }^{28,29}\right)$ reads $\partial_{x} F_{\mathrm{L} / \mathrm{T}}(\epsilon, x)=J_{\mathrm{L} / \mathrm{T}}(\epsilon)$, with $x$-independent energy, $J_{\mathrm{L}}$, and charge, $J_{\mathrm{T}}$, currents. Together with equation (1) this leads to an $x$-independent $F_{\mathrm{L}}(\epsilon)$ (for $|\epsilon|<\Delta_{0}$ ), satisfying the energy diffusion equation ${ }^{27} \partial_{\epsilon}^{2} F_{\mathrm{L}}=0$. As the selfconsistency relation

$$
\int_{\Delta(x)}^{\omega_{\mathrm{D}}} \mathrm{d} \epsilon \frac{F_{\mathrm{L}}(\epsilon, x)}{\sqrt{\epsilon^{2}-\Delta^{2}(x)}}=\int_{\Delta_{0}}^{\omega_{\mathrm{D}}} \mathrm{d} \epsilon \frac{\tanh (\epsilon / 2 T)}{\sqrt{\epsilon^{2}-\Delta_{0}^{2}}}
$$

( $\omega_{\mathrm{D}}$ is Debye frequency) involves only $F_{\mathrm{L}}$, it results in an almost constant $\Delta(x) \approx \Delta$. The phase slips in the middle of the wire excite quasiparticles and holes, equilibrating their populations. This provides the boundary condition $F_{\mathrm{L}}(|\epsilon|<\Delta)=0$ for the energy diffusion $^{27}$, resulting in the distribution function depicted in Fig. 3. On substitution into the self-consistency relation (2), it results in a transcendental equation for $\delta=\Delta / \Delta_{0}$, which at $T=0$ has two solutions (bistability): $\delta_{\mathrm{sc}}=1$ and $\delta_{\mathrm{vp}}=0.17$.

The second of these solutions implies a dissipative state with $e V_{0}=2 \Delta \approx 0.34 \Delta_{0}$. It is sustained if a normal current 


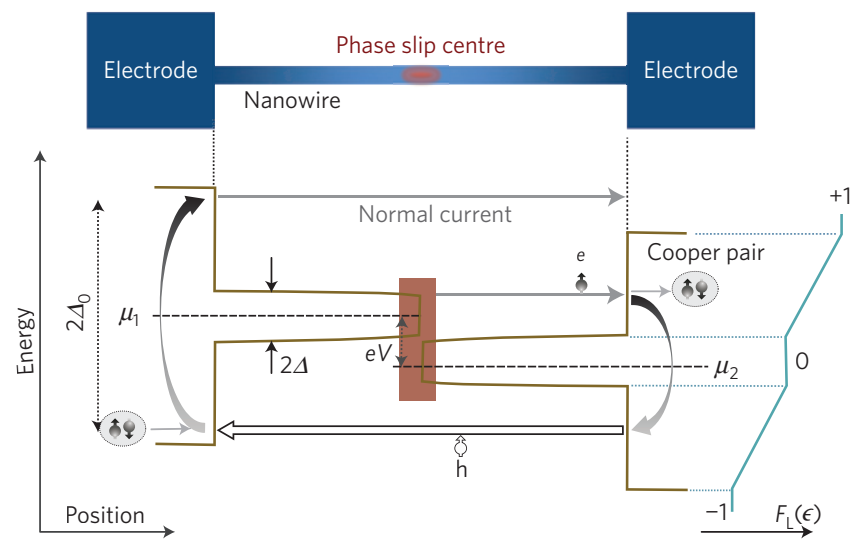

Figure 3 | Dissipative superconducting state of the nanowire. Top: Schematic of the system in which a superconducting nanowire is connected to superconducting leads. Bottom: The energy gap profile (solid brown) as a function of position along the system (horizontal direction). Phase slips occur in the centre of the wire (red region), generating non-equilibrium quasiparticles. At the two wire/lead interfaces they experience multiple Andreev reflections (shown schematically by blue-grey arrows) before escaping into the leads. This establishes a universal distribution, with the longitudinal part $F_{\mathrm{L}}(\epsilon)$ shown on the right. The self-consistency relation (2) results in the suppressed non-equilibrium gap $2 \Delta \approx 0.34 \Delta_{0}$, dictating that a voltage $e V_{0}=2 \Delta$ should develop to maintain phase slip events.

$I_{\mathrm{b}} \approx 1.64\left(V_{0} / R_{\mathrm{n}}\right)$ is applied to the wire (see Supplementary Methods). Note that for $\xi \ll L$ the current $I_{\mathrm{b}}$ is much smaller than the depairing critical current, allowing the wire to support a supercurrent. (This is different from long whiskers ${ }^{18}$, where phase slips extend over a few inelastic lengths, resulting in a negligible coherent supercurrent.) An excess current $I-I_{\mathrm{b}}$ is thus carried as the supercurrent, without an additional voltage increase-hence the observed voltage plateau. The current exceeding $I_{\mathrm{t}} \approx 0.72 \Delta_{0} / e R_{\mathrm{n}}$ stabilizes another solution of the self-consistency and energy diffusion equations: the one with a vanishing order parameter in the middle of the wire. It essentially terminates the supercurrent, resulting in the resistance being close to the normal-state resistance. The fact that these threshold values are approximately $30 \%$ less than those observed is attributed to residual inelastic processes, neglected above. Indeed, the latter lead to particle-hole recombination, driving the distribution towards the equilibrium one. A reasonable estimate $L_{\text {in }} \approx 12 \mu \mathrm{m}$ (ref. 17) brings the currents $I_{\mathrm{b}, \mathrm{t}}$ as well as the voltage plateau $V_{0}$ within $10 \%$ of the observed values.

This picture also naturally accounts for the observed effects of the magnetic field and temperature. The field mostly suppresses the order parameter of the leads $\Delta_{\text {lead }}$, leaving that of the wire (almost) intact. This narrows the interval for the energy diffusion ${ }^{30}$, bringing the distribution closer to the equilibrium one. This in turn increases the bottom threshold current $I_{\mathrm{b}}$. For $\Delta_{\text {lead }} \lesssim 0.78 \Delta_{0}$ the selfconsistent solution of Fig. 3 with $\delta \neq 1$ is no longer possible. The superconducting state of the wire is thus stabilized all the way up to $I_{\mathrm{t}}$. In fact, suppression of $\Delta_{\text {lead }}$ is also the primary mechanism of the voltage plateau termination at $T \gtrsim 650 \mathrm{mK}$ (Fig. 1b).

\section{Methods}

Investigated $\mathrm{Zn}$ nanowires were $100-120 \mathrm{~nm}$ wide, $65-110 \mathrm{~nm}$ high, with the length $1.5 \mu \mathrm{m}<L<6 \mu \mathrm{m}$ (Supplementary Table 1 ) connected to four $1-\mu \mathrm{m}$-wide $\mathrm{Zn}$ electrodes (inset to Fig. 1a). The $\mathrm{Zn}$ electrodes were $10 \mu \mathrm{m}$ long and in turn were connected to pre-patterned Au contacts. Both the nanowire and the electrodes were fabricated in a single step of the quench-deposition at substrate temperatures of $77 \mathrm{~K}$, depositing through a resist mask patterned using electron-beam lithography. The normal-state resistivity varies in the range $\rho=(6.2-8.4) \times 10^{-8} \Omega \mathrm{m}$ (in comparison the bulk value for $\mathrm{Zn}$ is $\left.\rho=5.9 \times 10^{-8} \Omega \mathrm{m}\right)$.
The electrical measurements were carried out in two different refrigerators. The first was a dilution refrigerator (Oxford Kelvinox-400) with a minimum temperature of $50 \mathrm{mK}$. The associated electrical lines were heavily filtered at room temperature using RC filters with a cutoff frequency of approximately $100 \mathrm{~Hz}$ and at low temperature using thermo coaxial cable filters with a cutoff frequency of approximately $1 \mathrm{GHz}$. The second was a Physical Properties Measurement System equipped with a He-3 insert (Quantum Design), with no filtering system other than having the electrical lines twisted in pairs.

To bypass effects of noise, the time-domain data was taken only in refrigerator 1 . The electrical measurements were performed with a bandwidth of $12 \mathrm{~Hz}$ and a repetition rate of $3 \mathrm{~Hz}$. At a fixed current in the transition regime, the voltage was continuously measured for $1,800 \mathrm{~s}$, exhibiting random switching in real time. To extract characteristic lifetimes of the superconducting $\left(\tau_{\mathrm{sc}}\right)$ and the voltage plateau ( $\tau_{\text {vp }}$ states the first $100 \mathrm{~s}$ of data was discarded to bypass possible transients. After that, whenever the voltage crossed above or below a threshold value (defined as three times the noise floor of the measurement set-up: $\sim 80 \mathrm{nV}$ ), the switching into or out of the voltage plateau state was recorded. The time interval between two consecutive switching events is defined as the residence time in one state. Finally, the values of $\tau_{\mathrm{sc}}$ and $\tau_{\mathrm{vp}}$ reported in the main text are mean values of the stochastic residence time sequence, collected over $1,800 \mathrm{~s}$.

Received 29 December 2013; accepted 27 May 2014; published online 29 June 2014

\section{References}

1. De Gennes, P. Superconductivity of Metals and Alloys 2nd edn (Westview Press, 1999).

2. Blatter, G. et al. Vortices in high-temperature superconductors. Rev. Mod. Phys. 66, 1125-1388 (1994)

3. Gol'tsman, G. N. et al. Picosecond superconducting single-photon optical detector. Appl. Phys. Lett. 79, 705-707 (2001).

4. Eisaman, M. D., Fan, J., Migdall, A. \& Polyakov, S. V. Invited Review Article: Single-photon sources and detectors. Rev. Sci. Instrum. 82, 071101 (2011).

5. Clarke, J. \& Wilhelm, F. K. Superconducting quantum bits. Nature 453, 1031-1042 (2008).

6. Devoret, M. H. \& Schoelkopf, R. J. Superconducting circuits for quantum information: An outlook. Science 339, 1169-1174 (2013).

7. Wollman, D. A., Van Harlingen, D. J., Lee, W. C., Ginsberg, D. M. \& Leggett, A. J. Experimental determination of the superconducting pairing state in YBCO from the phase coherence of YBCO-Pb dc SQUIDs. Phys. Rev. Lett. 71, 2134-2137 (1993)

8. Tsuei, C. C. et al. Pairing symmetry and flux quantization in a tricrystal superconducting ring of $\mathrm{YBa}_{2} \mathrm{Cu}_{3} \mathrm{O}_{7-\delta}$. Phys. Rev. Lett. 73, 593-596 (1994).

9. Mooij, J. E. \& Nazarov, Y. V. Superconducting nanowires as quantum phase-slip junctions. Nature Phys. 2, 169-172 (2006).

10. Bezryadin, A. Superconductivity in Nanowires: Fabrication and Quantum Transport 1st edn (Wiley, 2012).

11. Altomare, F. \& Chang, A. M. One-Dimensional Superconductivity in Nanowires 1st edn (Wiley, 2013).

12. Arutyunov, K. Yu, Golubev, D. S. \& Zaikin, A. Superconductivity in one dimension. Phys. Rep. 464, 1-70 (2008).

13. Sahu, M. et al. Individual topological tunnelling events of a quantum field probed through their macroscopic consequences. Nature Phys. 5, 503-508 (2009).

14. Shah, N., Pekker, D. \& Goldbart, P. M. Inherent stochasticity of superconductor-resistor switching behavior in nanowires. Phys. Rev. Lett. 101, 207001 (2008).

15. Li, P. et al. Switching currents limited by single phase slips in one-dimensional superconducting Al nanowires. Phys. Rev. Lett. 107, 137004 (2011).

16. Singh, M. \& Chan, M. H. W. Observation of individual macroscopic quantum tunneling events in superconducting nanowires. Phys. Rev. B 88, 064511 (2013).

17. Stuivinga, M., Mooij, J. E. \& Klapwijk, T. M. Current-induced relaxation of charge imbalance in superconducting phase-slip centers. J. Low Temp. Phys. 46, 555-563 (1982).

18. Skocpol, W., Beasley, M. R. \& Tinkham, M. Phase-slip centers and nonequilibrium processes in superconducting tin microbridges. J. Low Temp. Phys. 16, 145-167 (1974).

19. Chen, Y., Snyder, S. D. \& Goldman, A. M. Magnetic-field-induced superconducting state in $\mathrm{Zn}$ nanowires driven in the normal state by an electric current. Phys. Rev. Lett. 103, 127002 (2009).

20. Chen, Y., Lin, Y-H., Snyder, S. D. \& Goldman, A. M. Stabilization of superconductivity by magnetic field in out-of-equilibrium nanowires. Phys. Rev. B 83, 054505 (2011).

21. Tian, M. et al. Suppression of superconductivity in zinc nanowires by bulk superconductors. Phys. Rev. Lett. 95, 076802 (2005). 
22. Tian, M., Kumar, N., Wang, J., Xu, S. \& Chan, M. H. W. Influence of a bulk superconducting environment on the superconductivity of one-dimensional zinc nanowires. Phys. Rev. B 74, 014515 (2006).

23. Tidecks, R. Current-Induced Nonequilibrium Phenomena in Quasi-One-Dimensional Superconductors 1st edn (Springer, 1990).

24. Dinsmore, R. C. III, Bae, M-H. \& Bezryadin, A. Fractional order Shapiro steps in superconducting nanowires. Appl. Phys. Lett. 93, 192505 (2008).

25. Bae, M-H., Dinsmore, R. C. III, Sahu, M. \& Bezryadin, A. Stochastic and deterministic phase slippage in quasi-one-dimensional superconducting nanowires exposed to microwaves. New J. Phys. 14, 043014 (2012).

26. Barone, A. \& Paterno, G. Physics and Applications of the Josephson Effect 1st edn (Wiley, 1982)

27. Nagaev, K. E. Frequency-dependent shot noise in long disordered superconductor \normal-metal \superconductor contacts. Phys. Rev. Lett. 86, 3112 (2001).

28. Usadel, K. D. Generalized diffusion equation for superconducting alloys. Phys. Rev. Lett. 25, 507-510 (1970).

29. Kamenev, A. Field Theory of Non-Equilibrium Systems (Cambridge Univ. Press, 2011).

30. Vodolazov, D. Y. \& Peeters, F. M. Enhancement of the retrapping current of superconducting microbridges of finite length. Phys. Rev. B 85, 024508 (2012).

\section{Acknowledgements}

Discussions with X. Wang are warmly acknowledged. Experimental work at Minnesota was supported by the DOE Office of Basic Energy Sciences under Grant No.

DE-FG02-02ER4600. Samples were fabricated in the Nano Fabrication Center, which receives funding from the NSF as a part of the National Nanotechnology Infrastructure Network, and were characterized in the Characterization Facility, University of Minnesota, a member of the NSF-funded Materials Research Facilities Network (http://www.mrfn.org) via the MRSEC program. AK was supported by DOE Contract No. DE-FG02-08ER46482

\section{Author contributions}

Y.C. and A.M.G. conceived and designed the experiment. Y.C. and S.D.S. fabricated the devices. Y.C and Y-H.L. performed the measurements. A.K. provided theoretical analysis and co-wrote the manuscript with Y.C. and A.M.G. All authors contributed to the discussion and presentation of the results.

\section{Additional information}

Supplementary information is available in the online version of the paper. Reprints and permissions information is available online at www.nature.com/reprints.

Correspondence and requests for materials should be addressed to A.M.G.

\section{Competing financial interests}

The authors declare no competing financial interests. 